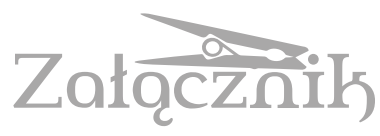

\title{
TRANSPARENCY AS A FUNCTIONAL AND VISUAL TOOL. THE GUI OF THE WINDOWS 10 OPERATING SYSTEM AS AN EXAMPLE
}

AGNIESZKA SMAGA

Wydział Nauk Humanistycznych UKSW

Faculty of Humanities, Cardinal Stefan Wyszyński University in Warsaw

a.smaga@uksw.edu.pl

Transparency ${ }^{1}$ mediates between a body and light, and the surrounding multidirectional reality, and then their observer, that is, at the meeting point of various physicalities. Thus, it has an ambiguous ontic status. It is also the property of a material and its surroundings and the effect of their perception and cognition (see: Błaszczak 2016). At this point we can recall the somewhat corrected maxim of Frank L. Wright that 'every material (A.S. also all of its properties) carries its own message', thus opening the field for further discussion and analysis of the category of transparency (Wright 1975: 171). Transparency can be analysed in many of its dimensions, such as philosophy, aesthetics, ethics, art, utility, performance, law, sociology and psychology. Thus, transparency functions within, or rather on the edges of many research disciplines, including natural, humanistic, social, medical and technical ones. Its interdisciplinarity does not, therefore, need to be scientifically proven. Transparency should therefore be treated based on categories, understood in accordance with mathematical (Wiweger 1993; Turi 1996-2001) formula, i.e. viewed only initially from the inside (locally), but above all from the outside (globally), through its behaviour in relation to other elements behind and in front of it, as well as next to it, on top and at the bottom. Thus, the category of transparency, like weight, speed and others, becomes, principium comparationis, an area and a tool for modern,

1 The category of transparency has a physical origin and is defined as the ability of a material or medium to transmit light. 
intermedial comparative studies. ${ }^{2}$ This discipline nowadays focuses not on artifacts (as in mathematical category theory - not elements of a set or sets), but relations between them. Therefore, the object of scientific reflection is replaced by the process, presentation by communication, linearity by nonlinearity and contemplation by interaction.

Transparency becomes a dynamic, changing element of the relationship, a layer functioning between the space of the transparent body and the surrounding reality, including human space. Therefore, transparency is a medium that mediates information in a specific way. Being the means of transmission - a surface, an appearance - it postulates the existence of something more than itself. Transparency suggests an existence of a hidden depth which adds a mysterious and magical dimension. Transparency exists mainly at the surface level (signifiant), within arm's reach, thus it is controllable. Unlike the depth of the message (signifié), which, when obscured, creates an inaccessible, hidden impression. Physical-perceptual transparency thus turns out to be aesthetically attractive and functionally practical. Therefore, this physical property is also an aesthetic and pragmatic category, like speed, weight and lightness.

These properties of transparency and the suggested visual-functional perspective mean that transparency in the digital environment becomes a medium with the laws of technology applied to it - as a n activity and a tool for effective communication and use. It is therefore considered as an operational category - accompanying the process of generating and using a message - and an instrument - supporting software. Both types of transparency mutually condition each other at different levels of the digital world - operating systems, applications, websites, search engines and web browsers, etc. The transparency of operation (of an operational and functional nature) is based on the automatic launch of 'hardware or software mechanisms, in such a way that the user does not know and does not need to know about the specific methods used' (Lathman 1997: 163) 3 . This kind of transparency, despite its nature described above, blurs or even deprives

2 Similarly, Monika Błaszczak (2016) noted and partially described the properties of this category.

3 Operational transparency is particularly important in the case of a distributed system and it means that the user perceives it as a whole and not as a set 
users of the need to look deeper into knowledge about IT and hardware ${ }^{4}$. In the mental dimension, the transparency of operation makes the tool itself transparent, which results in perceptual transparency and, as a result, in not recognising the material of the instrument itself. Screen transparency has a narrow definition in design as a 'visual effect, associated with an object partially obstructing what is behind it. This effect can be rendered using analytical calculations as part of the ray tracing method or based on weight averaging when using the painting method' (Lathman 1997: 162; Traczyk 2010). In this case, transparency is treated literally as a visual feature, modelled on the physical properties of the material. Understood in this way, it becomes a tool within a large set of digital instruments available for the g r a phical user interface (GUI) that supports various levels of the digital world, such as operating systems, user applications, websites, mobile apps and web browsers. Transparency is a key feature of graphical interfaces, especially present in the Windows 10 operating system update launched at the end of April 2018 ${ }^{5}$. This category enables Windows 10 to enter the era of mixed reality and holographic processing ${ }^{6}$. The April update initiated a new style of graphical user interface for the system and applications called Fluent

of individual components. https://www.szkolnictwo.pl/szukaj,System_rozproszony [access date: 10.07.2018].

4 In order to fulfill the functions assigned to it, the tool cannot be physically and functionally transparent, although it has often been treated that way in literary and cultural studies. This is probably because objects treated as useful (as opposed to artistic), namely tools, today's digital graphics, became after their implementation something so obvious that they seemed transparent, thus research attention was not focused on them.

5 This modernisation was previously known as Spring Creators Update, and for supporters of code names - just Redstone 4.

6 Mixed reality combines augmented virtuality (AV) with virtual reality (VR) and augmented reality (AR). The first, augmented virtuality, introduces virtuality support using physical stimuli (e.g. head movements using a gyroscope on a smartphone) or dynamically inserts fragments of reality into a virtual area (e.g. some games on Microsoft Kinect and Playstation Move). The second, virtual reality, is created by computer and has no physical form. The third, augmented reality, makes it possible to superimpose onto reality layers of $2 \mathrm{D}$ and $3 \mathrm{D}$ digital objects, scanned with a camera, with which the user enters limited interaction. 
Design, which replaced the previous Project Neon ${ }^{7}$. The new style was dictated by aesthetic, but above all, functional considerations. Both reasons for the proposed changes are mutually integrated in the graphical user interface. The system's visually updated and user-friendly system GUI aims to connect with other very different devices: Android ${ }^{8}$ phones and tablets, as well as computers or even mixed-reality glasses. The implementation of the new Windows design language was launched initially based on previous interfaces of applications, such as Music Groove, Post and Film \& $\mathrm{TV}$, but its aim is to introduce comprehensive liquefaction, that is, to refresh the entire graphics of the system. The consistency of the Windows operating system on different devices and in mixed reality (a combination of virtual, real, and augmented) is to be achieved by technically and visually 's m o o th ' transitions between the specified functional components (hardware and the digital and physical environment). The effect of liquid, scalar transitions is obtained by adding five new aspects to the current 'flat' interface style: light, depth, motion, material and scale. All the GUI properties listed above are also associated with transparency understood as the physical property of an object. For the existence of this property, light is required to pass through the transparent surface of a material, which covers and reveals the depth of subsequent layers and with its variable scalability plays a game between the viewer and the world. Observed convergence suggests that an imitation of the physical effect of transparency will enable virtual and extended worlds to 'smoothly' blend into reality'.

These artificial elements are static, i.e. they cannot be rotated, minimised or moved as in the physical world.

7 https://www.microsoft.com/design/fluent/ [access date: 10.07.2018].

8 Installing the Microsoft Launcher application allows users to personalise devices by applying refreshed daily wallpapers, theme colours, leading colours and icon packs. When users take a photo on their phone, they can view it instantly on their computer or continue editing an Office 365 document on their computer by connecting their phone to a Windows computer.

9 For this to happen, skeumorphism is making a return. 


\section{Microsoft Fluent Design System}

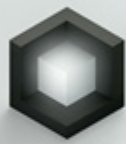

Light

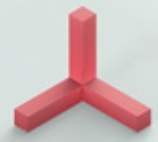

Depth

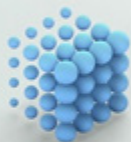

Motion

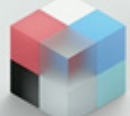

Material

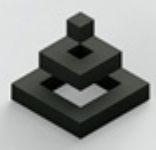

Scale

Figure 1. Fluent Design components ${ }^{10}$

Therefore, what Fluent Design builds is a visual impression of depth ${ }^{11}$. This effect, most clearly visible in the calendar application, introduces the principles of convergent, air and colour perspective, as well as grades of shade, colour and transparency. The illusion of depth is achieved primarily through layers, which means that planes can be manipulated freely (for example in Windows Ink). In this case, transparency categories allow us to look through one window and see simultaneously the layout of other windows ${ }^{12}$.

${ }^{10}$ https://www.microsoft.com/design/fluent/ [access date: 10.07.2018].

11 The new design is a bit like what OS X Yosemite introduced to the Apple system.

12 In the first graphical interfaces of operating systems, the transparency effect was only suggested and appeared on the edge of windows as they overlapped. The (title, status and scroll) bars of interface windows were opaque, but placed on top of each other they created an impression, which corresponds well to the title category, of transparency and a participation of first, second and third levels, or depth. Illusion gives birth to illusion, and this brings another to life. The suggested transparency effect was less real and obvious than the one introduced by the visualization of the physical properties of glass, which is why it is certainly stimulating 
The next change concerns the aspect of the material and refers to the elements, imitating matted glass. The acrylic function is responsible for this effect, which is the first and at the moment the only one used by Fluent. Each newly introduced material will most likely mean a new form and new use (Wright 1975: 171).

Microsoft does not call the new style just a design language, it calls it a design system, because it introduces visual effects that activate new modes of use. This innovative practice takes place in Windows Mixed Reality, sometimes called a hybrid reality ${ }^{13}$. MR combines the experience of VR, $\mathrm{AR}$ and $\mathrm{AV}$.

Mixed reality, also known as hybrid reality - in fact is a combination of the three concepts described above - that is, the virtual world with the real one so that virtual and real objects interact dynamically. In contrast to reality and augmented virtuality, we are not dealing here with placing objects from one world into another, only mixing real and virtual worlds (ViReal 2015).

MR determines the whole spectrum of forms and interactions that operate between virtual reality and augmented reality. It allows the virtual world to be mapped in the real world, mixing them together using HoloLens goggles (or other similar devices) along with controllers for applications. With HoloLens glasses equipped with a camera and sensors, the user can scan the surroundings and create a three-dimensional model on which virtual content will be placed. This happens using mixed reality motion controllers that allow natural interaction without installing additional devices on walls.

The Windows operating system and the style of its visualisation satisfy quite a few desires, for example from 2002: 'In a dream living room, the TV and the music equipment are not separate but interact with each other, with the Internet, the PC in the home office or with electronic games in the play

perceptively and above all mentally. This transparency created the environment of structural, spatial looking 'at' and 'through' visual forms. It stimulated the user to actively and mentally build imagined constellations.

13 To do this, in order to display the Start menu and continue to operate the system and applications in real life, users press the Windows button on the controller or say 'Go to Start'. 
room' (CEA 2002). Modern operating systems allow the hardware described above and other devices such as smartphones and tablets to integrate with each other. They even allow utility software (text, image, document and email editors) and entertainment software (the illusory reality of 3D computer games, free and paid, Minecraft, Galaxy Explorer and others, or 'immersion' in watching movies in 360) to be displayed. And this display can be directly on the wall in the 'dream living room'. The new operating system also supports the creative process by enabling the introduction to MR of readymade or self-made 3D objects in Paint 3D.

The operation of elements from virtual worlds in mixed reality will reflect the user's experience, known from the physical world, i.e. objects can be moved, resized, zoomed in or out, 3D objects can be moved and rotated at different rates and by five animation models. Websites or application windows can be scrolled just like on a computer, but this time on a wall. Users can also take photos in mixed reality and send them to the virtual reality of a computer.

In the real world, the window frame tells us about the transparency of the window. Physical transparency is perceived through contrast with an opaque element and is thus a game between surface and depth, becoming a component of one or the other or both. It is therefore a property of surface and depth and our knowledge of them. There is no depth without transparent glass enclosed in a window frame, and vice versa - there is no transparency effect without the world 'showing through'. In Windows Vista, only the bars (title, task, status, scroll bars) were translucent and were perceived by their contrast with the opaque content of the system window or application, thus slowly blending into the virtual environment. Now these elements are liquefied in the real world. Now, even window contents - metaphorical glazing - use scales of transparency. Various data from many media and worlds are displayed, not on the screen, but directly on a wall, for example in the living room. The physical window-screen screen - which, like the window frame in a real window is visible and stable - now slowly disappears. Digital data is provided bypassing the form of the screen, but not bypassing the eye or the optics of seeing, as in neural interfaces. All that remains are semi-transparent interface strips and to a variable degree transparent window contents. 


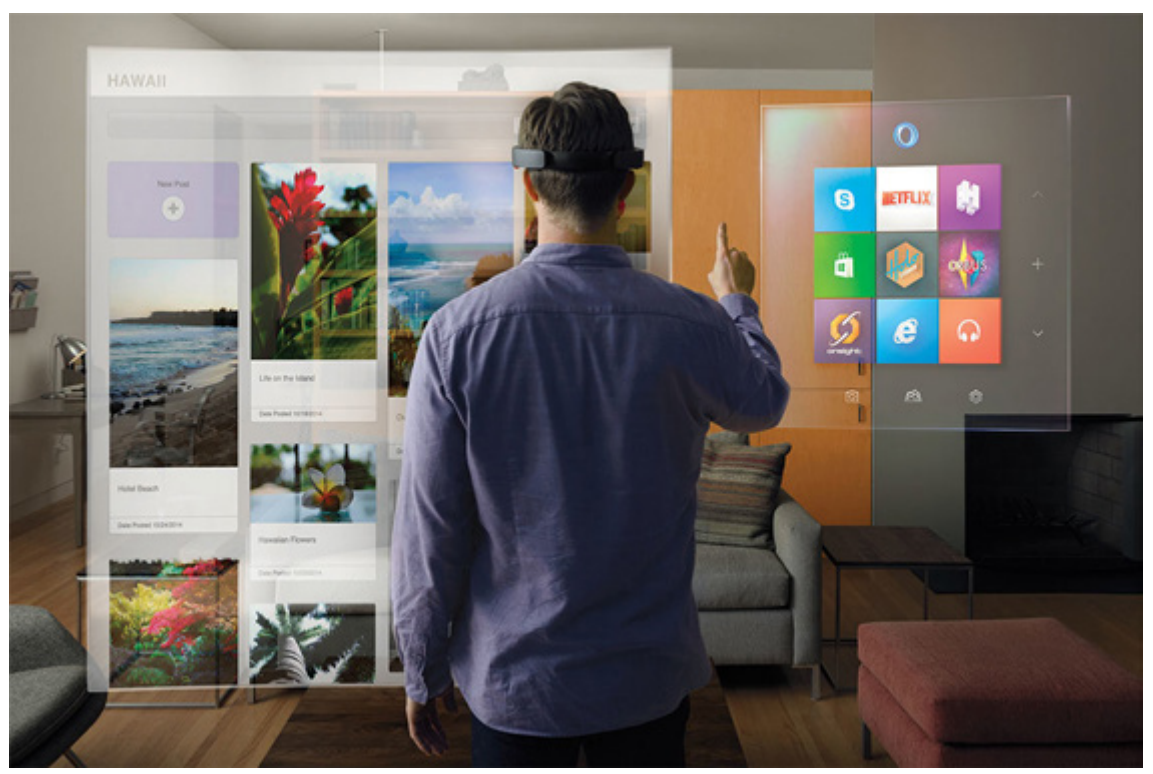

Figure 2. Mixed reality ${ }^{14}$

Mixed worlds use and mix two models of reality, and thus transparency. Transparency in virtual reality helped to simulate the three dimensions of the game world presented in two dimensions of the screen and thereby introduced depth effects as if viewed through a window pane. The transparency in this case imitated the physical property of material letting light through it and allowed users to immerse themselves in what was being presented. However, transparency treated as an interface element built the illusion of three dimensions, but sometimes it remained flat. It offered a permeability that did not focus attention on itself and encouraged users to interact comfortably, economically and intuitively with digitally created worlds. Transparency in this case allows game players to look 'discreetly' at the GUI and to see and operate worlds presented in a game.

14 https://www.amicopc.com/wp-content/uploads/2017/06/windows-10 -mixed -reality-1024x682.jpg [access date: 10.07.2018]. 
Transparency in augmented reality has also played a very important role. It allows static and dynamic $2 \mathrm{D}$ or $3 \mathrm{D}$ worlds generated by computer to be skilfully applied to a three-dimensional world, scanned using cameras embedded in translucent glasses. Transparency lays subsequent layers in a way that allows users to perceive themselves and real space at the same time, and allows content to be optimised. Literal transparency connected with looking 'through' the window, as well as the phenomenal transparency of looking 'at' the window resulting from the structural environment of looking appear simultaneously.

Mixed worlds build compiled models and construct even more advanced game rules with the user. MR participants see and use $2 \mathrm{D}$ or 3D simulation images from virtual, augmented and real worlds of a static, dynamic or interactive nature, captured at equal angles, sequentially, multicast, linearly, constellationally or by hypertext. All these images are superimposed on each other by scalable transparency. The windows, in fact only their panes, overlap giving the effect of three-dimensional surfaces (previously they were flat), ready for them to be re-arranged, structured on the three dimensions of the real world (previously it was two desktop sizes). Multiphase transparency helps in the multiplication and smooth transition of these planes, facilitating movement backwards and forwards between programs and documents. This occurs in many of its saturations, called waves, so it is better to speak about a lot of transparency in this case, which corresponds to the scalar nature of this category. Transparencies lead games between their states, then between realities (real, augmented, virtual), between the size of the windows and the elements operating them. They build dynamic, variable structures of presentation, different in different configurations, depending on the type, number and order of windows, programs and applications. Views cannot be repeated. Users look with difficulty 'at' the digital view, as they used to at the interface window, more and more often they perceive 'through' the view, analogically as through a window pane. 


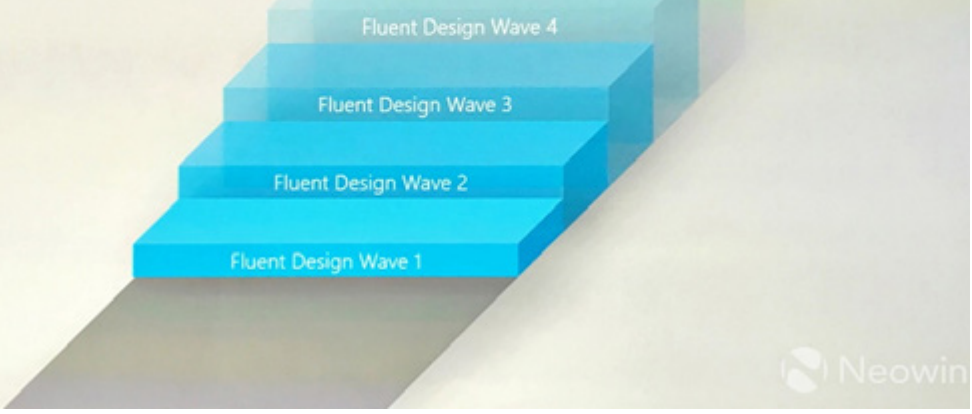

Figure 3. Transparency system ${ }^{15}$

Transparencies enter real space to allow it to be better integrated with virtual space. Both worlds begin to permeate, just like layers in graphics programs. The boundary between the physical wall and the virtual presentation (with hidden content) and the shell (the graphical interface that slowly disappears in its visual dimension, but not in its operational aspect) and between the display is blurred. The elements of the interface that support virtual content become messages themselves. Space is dynamised by the spatialisation of time. Mixed reality, like the category of transparency, functions between different physicalities and has an ambiguous ontic status. Transparencies are involved in creating mixed reality, total space, an authentic graphic work environment, entertainment and leisure. Multiplied surfaces and fewer windows make computer multitasking possible.

On the one hand there are multi-layered transparencies of views, while on the other there are transparencies for supporting views, real world transparencies and multitasking. All these factors give the user a lower level of immersion in technology than in the case of VR, but greater than

15 https://s3.amazonaws.com/neowin/news/images/uploaded /2017/05/1494545330_fluent_2.jpg [access date: 10.07.18]. 
in AR. They also provide a sense of dispersion of subjectivity and a high degree of effective interactivity.

In summary, the last version of Windows offers various forms of GUI transparency at the hardware level in terms of operating, and at the software level in terms of functionality and aesthetics, where aesthetics is connected with the literal visualisation effect and the mental building of structures. The transparent digital views overlapping each other to different degrees become a tool for the organisation of space, the recipient's structural vision and, above all, use. Transparencies are introduced within the contents of a window, but above all within elements that support virtual content. They conduct, at various levels of their saturation, a kind of multi-level game: between their form and the content hidden behind it, visible and invisible, an illusion and a disillusion, materialisation and dematerialisation, selfreality and reality existing beyond it, finally between itself and recipients, forcing them to specific perceptions, reactions, behaviours, and valuations.

\section{Selected bibliography}

Architecture and Cubizm (2002), ed. N.J. Troy, E. Blau, Cambridge (MA): MIT Press. Rudolf Arnheim (1974), Art and Visual Perception, Berkeley: University of California Press.

Monika Błaszczak (2016), Przezroczysta lekkość bytu - od Calvina do Bieńczyka, „Przestrzenie Teorii”, no. 25.

Jay David Bolter (2001), Writing Space: The Computer, Hypertext, and the Remediation of Print, Mahwah: Lawrence Erlbaum Associated Inc.

Anna Friedberg (2006), The Virtual Window: From Alberti to Microsoft, Cambridge (MA): The MIT Press.

Steven Johnson (1999), Interface Culture: How New Technology Transforms the Way We Create and Communicate, New York: HarperCollins.

Roy Latham (1997), Leksykon grafiki komputerowej i rzeczywistości wirtualnej, trans. J. Zabrodzki, Warszawa: Wydawnictwa Naukowo-Techniczne.

Lev Manovich (2001), The Language of New Media, MIT Press, Cambridge (MA). Andrzej Wiweger (1993), Teoria kategorii, [in:] Leksykon matematyczny, ed. M. Kordos, M. Skwarczyński, W. Zawadowski, Warszawa: Wiedza Powszechna. 
Frank Lloyd Wright (1975), VI. Th Meaning of Materials - Glass, [in:] In the Cause of Architecture, ed. F. Gutheim, New York: Architectural Record.

\section{Digital sources}

https://www.dobreprogramy.pl/Project-Neon-to-Fluent-Design-nowy-wyglad-

Windowsa-na-Build-2017,News,80991.html.

https://www.microsoft.com/design/fluent/.

https://www.microsoft.com/pl-pl/windows/windows-ink.

https://www.spidersweb.pl/2018/05/windows-10-fluent.html.

https://www.szkolnictwo.pl/szukaj,System_rozproszony. http://zs9elektronik.pl/

naszeprace/raftra/skryptgk.pdf.

Daniel Turi (1996-2001), Category Theory Lecture Notes., http://www.dcs.ed.ac.uk/ home/dt/CT/categories.pdf.

ViReal (2015), Microsoft Hololens - inny typ rzeczywistości, https://vrpolska.eu/ bez-kategorii/microsoft-hololens-inny-typ-rzeczywistosci/.

\section{Transparency as a Functional and Visual Tool. The GUI of the Windows 10 Operating System as an Example}

Transparency mediates between a body and light, and the surrounding multidirectional reality, and then their observer, that is, at the meeting point of various physicalities. Transparency suggests an existence of a hidden depth which adds a mysterious and magical dimension. Transparency exists mainly at the surface level (signifiant), within arm's reach, thus it is controllable. Unlike the depth of the message (signifié), which, when obscured, creates an inaccessible, hidden impression.

Transparency in the digital environment becomes a medium with the laws of technology applied to it - as an activity and a tool for effective communication and use. Transparency is therefore considered as an operational category - accompanying the process of generating and using a message and an instrument - supporting software. Both types of transparency mutually condition each other at different levels of the digital world - operating systems, applications, websites, search engines and web browsers, etc. The last version of Windows offers various forms of GUI transparency at the hardware level in terms of operating, and at the software level in terms of functionality and aesthetics, where aesthetics is connected with the literal 
visualisation effect and the mental building of structures. Transparencies conduct, at various levels of their saturation, a kind of multi-level game: between their form and the content hidden behind it, visible and invisible, an illusion and disillusion, materialisation and dematerialisation, self-reality and reality existing beyond it, finally between itself and recipients, forcing them to specific perceptions, reactions, behaviours, and valuations.

Keywords: applied graphics, comparative media studies, transparency of operation, screen transparency, Graphical User Interface (GUI), Fluent Design, Mixed Reality, User Experience (UX) 